\author{
David F. Gaieski \\ Marie E. Beylin \\ Benjamin S. Abella \\ Anne V. Grossestreuer \\ Sarah M. Perman
}

\section{What is the optimal post-arrest hemodynamic strategy? Towards personalized resuscitation strategies}

Accepted: 13 December 2013

Published online: 14 January 2014

(C) Springer-Verlag Berlin Heidelberg and ESICM 2013

Dear Editor,

Thank you for the opportunity to reply to Drs. Dell'Anna and Taccone's letter [1] commenting on our recent article entitled "Higher mean arterial pressure with or without vasoactive agents is associated with better survival and neurological outcomes in comatose survivors of cardiac arrest" [2]. The fundamental insight of our research was that higher mean arterial pressure (MAP) during the first day post-arrest was associated with better survival and neurological outcomes than lower MAP.

Overall, we agree with Drs. Dell'Anna and Taccone's thoughtful analysis. They correctly point out that we combined agents with very different mechanisms of action into a general category of "vasoactive" agents. However, a subgroup analysis, not reported in the manuscript, limited to traditional vasopressors and inotropes yielded similar results. Further, we agree that prospective analyses of vasopressor burden, i.e., the dose and duration a patient requires, and vasopressor timing, i.e., early vs. late administration, are needed to gain better insight into the contributions shock, myocardial stunning, and the sepsis-like syndrome make to post-arrest outcomes.

Better yet would be a trial of postarrest care with patients randomized to varying MAP goals: with adequate cardiac and cerebral monitoring, insights into the fine balance between cerebral perfusion and myocardial stress may emerge. The authors point out Bouzat et al.'s recent research demonstrating that increasing MAP from 70 to $90 \mathrm{mmHg}$ in comatose post-arrest patients treated with therapeutic hypothermia (TH) did not improve cerebral perfusion. However, blood pressure manipulation was performed on average $17 \pm 5 \mathrm{~h}$ after arrest, during the stable maintenance phase of TH. In contrast, our most important findings were at hours 1 and 6 , when post-arrest hemodynamics are rapidly changing and have not reached a "stable maintenance phase."

In our opinion, informed by the work of Rittenberger et al., there are several categories of post-arrest patients, roughly categorized by the combined degree of cerebral and cardiac dysfunction. Optimal postarrest management will only occur when real-time quantification of the degree of organ injury becomes available and therapy can be "personalized" to the individual patient's injury pattern with optimal hemodynamics augmented by surrogates of tissue perfusion including central venous oxygen saturation, lactate clearance, urine output, cerebral oxygenation, cardiac output, and echocardiography. Indeed, in the Bouzat et al. study, some patients had increased and others had decreased cerebral tissue oxygenation in response to induced hypertension. These patients are demonstrating different injury patterns requiring different therapeutic interventions.

Finally, we agree that granular information about multi-organ system dysfunction and cause of death would provide further insight into the relationship between MAP and outcomes.
In prior studies we have demonstrated large clinician-level variability in withdrawal of care and patient-level variability in time to awakening after cardiac arrest. These data were not available in our retrospective analysis and should be collected in future prospective observational studies. We encourage other researchers to perform these difficult but worthwhile studies. Despite marked improvements in post-arrest outcomes over the past decade, there remains much room for improvement and a key to further improvement is hemodynamic optimization.

Conflicts of interest No conflict of interest.

\section{References}

1. Dell'Anna AM, Taccone FS (2013) Target mean arterial pressure after cardiac arrest. Intensive Care Med. doi: 10.1007/s00134-013-3161-z

2. Beylin ME, Perman SM, Abella BS, Leary M, Shofer FS, Grossestreuer AV, Gaieski DF (2013) Higher mean arterial pressure with or without vasoactive agents is associated with better survival and neurological outcomes in comatose survivors of cardiac arrest. Intensive Care Med 39:1981-1988. doi: $10.1007 / \mathrm{s} 00134-013-3075-9$

D. F. Gaieski $(\bowtie) \cdot$ B. S. Abella •

A. V. Grossestreuer

Center for Resuscitation Science, Department of Emergency Medicine,

Perelman School of Medicine at the University of Pennsylvania, 34th and Spruce Streets, Ground Ravdin, Philadelphia, PA 19104, USA

e-mail: David.Gaieski@uphs.upenn.edu Tel.: +1-215-3495241; +1-302-5887083

Fax: +1-215-6623953

M. E. Beylin

Department of Emergency Medicine, San Francisco School of Medicine, University of California, San Francisco, CA, USA

\section{S. M. Perman}

Department of Emergency Medicine, University of Colorado School of Medicine, Aurora, CO, USA 